\title{
Environmental and occupational exposure to benzene by analysis of breath and blood
}

\author{
L PERBELLINI, ${ }^{\prime}$ G B FACCINI, ${ }^{2}$ F PASINI, ${ }^{2}$ F CAZZOLI, ${ }^{3}$ S PISTOIA, ${ }^{3}$ \\ R ROSELLINI, ${ }^{3}$ M VALSECCHI, ${ }^{4}$ F BRUGNONE ${ }^{1}$ \\ From the Istituto di Medicina del Lavoro dell'Università di Verona, ${ }^{1}$ Istituto di Chimica e Microscopia Clinica \\ dell'Università di Verona, ${ }^{2}$ Montedipe Mantova, and Unita Locale Socio Sanitaria, 47 Mantova, Italy
}

\begin{abstract}
Benzene exposure of chemical workers was studied, during the entire workshift, by continuous monitoring of workplace benzene concentration, and 16 hours after the end of the workshift by the measurement of alveolar and blood benzene concentrations and excretion of urinary phenol. Exposure of hospital staff was studied by measuring benzene concentrations in the alveolar and blood samples collected during the hospital workshift. Instantaneous environmental air samples were also collected, at the moment of the biological sampling, for all the subjects tested. A group of 34 chemical workers showed an eight hour exposure to benzene, as a geometric mean, of $1 \cdot 12 \mu \mathrm{g} / \mathrm{l}$ which corresponded, 16 hours after the end of the workshift, to a geometric mean benzene concentration of $70 \mathrm{ng} / \mathrm{l}$ in the alveolar air and $597 \mathrm{ng} / \mathrm{l}$ in the blood. Another group of 27 chemical workers (group A) turned out to be exposed to an indeterminable eight hour exposure to benzene that corresponded, the morning after, to a geometric mean benzene concentration of $28 \mathrm{ng} / \mathrm{l}$ in the alveolar air and $256 \mathrm{ng} / 1$ in the blood. The group of hospital staff (group B) had a benzene concentration of $14 \mathrm{ng} / \mathrm{l}$ in the alveolar air and $269 \mathrm{ng} / \mathrm{l}$ in the blood. Instantaneous environmental samples showed that in the infirmaries the geometric mean benzene concentration was $58 \mathrm{ng} / \mathrm{l}$ during the examination of the 34 chemical workers, $36 \mathrm{ng} / \mathrm{l}$ during the examination of the 27 chemical workers (group A), and $5 \mathrm{ng} / \mathrm{l}$ during the examination of the 19 subjects of the hospital staff (group B). Statistical analysis showed that the alveolar and blood benzene concentrations in the 34 workers exposed to $1 \cdot 12 \mu \mathrm{g} / 1$ of benzene differed significantly from those in groups A and B. It was found, moreover, that the alveolar and blood benzene concentrations were higher in the smokers in groups $\mathrm{A}$ and $\mathrm{B}$ but not in the smokers in the group of 34 chemical workers. The slope of the linear correlation between the alveolar and the instantaneous environmental benzene concentrations suggested a benzene alveolar retention of about $55 \%$. Blood and alveolar benzene concentrations showed a highly significant correlation and the blood/air partition coefficient, obtained from the slope of the regression line, was $7 \cdot 4$. In the group of the 34 chemical workers no correlation was found between the TWA benzene exposure and the urinary phenol excretion.
\end{abstract}

Exposure to benzene may induce leukaemia in man,' and at present a lowering of its hygienic environmental limits is suggested by many national and international authorities. Italian law forbids the use of benzene as an industrial solvent and allows it to be used only when its replacement with a suitable compound is chemically impossible.

In this practical context it was our aim to see if it is possible to carry out biological monitoring of workers exposed to benzene by measuring benzene in the

Accepted 6 April 1987 alveolar air and blood collected 16 hours after the end of the workshift. The numerous data on the biological monitoring which have been reported previously were recently reviewed in a document of the Commission of the European Communities. ${ }^{2}$ Frequently, these data refer to benzene exposure concentrations higher than $10 \mathrm{ppm}(30 \mu \mathrm{g} / \mathrm{l})$ and to investigations carried out on volunteers experimentally exposed under standard laboratory conditions. In our research we studied exposure to benzene by comparing occupationally exposed workers with non-occupationally exposed individuals. 


\section{Materials and methods}

With reference to exposure to benzene 80 subjects, split into three groups, were examined. The first group included 34 workers employed in the production of benzene in a chemical plant (workers). The second group included 27 workers of the same plant but not employed in the production of benzene (group A). The third group included 19 subjects belonging to the staff of a university hospital (group B) located in a different town from that of the chemical plant.

With regard to the individual workers of the chemical plant the exposure to benzene was monitored continuously during the eight hours of the workshift by means of charcoal tubes and portable pumps. The morning after the monitored workshift, the workers were examined in the infirmary of the plant by collecting one sample of alveolar air, blood, and urine. At the same time as the alveolar and blood samples, one instantaneous sample of the infirmary environmental air was collected for each worker.

With regard to the individuals of the hospital staff (group B) the examinations were carried out in the infirmaries of the hospital wards. For each subject, one alveolar and one blood sample were collected at an unspecified time of the day. At the same time, one instantaneous sample of the infirmary environmental air was collected for each subject.

AIR SAMPLING

Continuous environmental samples were collected for the entire workshift (eight hours) by means of portable pumps and charcoal tubes to estimate the exposure of the chemical workers both employed and not employed in the production of benzene. The sampling flow rate was $11 / \mathrm{min}$. Gas chromatographic determination of benzene was carried out according to the NIOSH method. ${ }^{3}$

In the infirmaries instantaneous environmental air samples were taken from the breathing zone of each individual at the place he was during the alveolar and blood sampling. Environmental samples were collected in stoppered glass tubes with screw caps at both ends and an inside volume of $250 \mathrm{ml}$, by manual pump.

Alveolar air samples were collected in glass tubes similar to those used for environmental air. Each individual subject after a normal inspiration gave a forced expiration keeping the glass tube between the lips. The tube was immediately sealed with the two caps after the end of the expiration.

\section{BLOOD SAMPLING}

Ten millilitres of venous blood taken from each individual were injected into $12 \mathrm{ml}$ glass vials containing one drop of ethylenediaminetetra-acetate (EDTA) solution and provided with a screw cap. For the analysis in the laboratory $5 \mathrm{ml}$ of the blood sample were injected into the $250 \mathrm{ml}$ glass tubes described above. The glass tubes were put in a hot room at $37^{\circ} \mathrm{C}$ for at least one hour and the blood analyses were carried out by means of a head space technique.

Before sampling, all the glass tubes used for environmental, alveolar, and blood analyses were kept for one night in an oven at $100^{\circ} \mathrm{C}$ and then washed with a flow of "zero degree air" for five minutes.

URINE SAMPLING

Only the workers employed in the production of benzene provided a sample of urine in the morning and at the end of the workshift. Urinary phenol was measured by the colorimetric method using 4-aminoantipyrine as a reagent. Urinary phenol concentrations were adjusted at the specific gravity of 1024 .

\section{GAS CHROMATOGRAPHY}

The glass tubes were connected by a six port valve to a loop of $50 \mathrm{ml}$. The air contained in the glass tubes was transferred to the loop by a gentle suction of about $100 \mathrm{ml}$. Subsequently, the contents of the loop were transferred to a cryogenic trap made as follows: a stainless steel tube of $10 \mathrm{~cm}$ with an internal diameter of $0.3 \mathrm{~mm}$ and with a $U$ bend containing $3 \mathrm{mg}$ of Tenax in the central portion. The Tenax trap was kept at $-10^{\circ} \mathrm{C}$ in an ethylene glycol bath during the air sample transfer from the loop to the trap. After the loading of the cryogenic trap a splitter valve was closed and the air flow was connected only with the capillary column of a gas chromatograph. The transfer from the trap to the capillary column was made by a flash heating $\left(300^{\circ} \mathrm{C}\right.$ in 2.5 seconds) of the trap which was connected to an electrical circuit of three volts and 40 amperes.

Apart from the cryogenic trap, all the tubing from the loop to the connection with the capillary column was maintained at $80^{\circ} \mathrm{C}$ to avoid solvent condensation on the tube walls.

A Hewlett Packard 5890 gas chromatograph equipped with silica capillary column of cross linked $5 \%$ phenyl-methyl-silicone $0.17 \mu$ thickness, $50 \mathrm{~m}$ long, and with $0.32 \mathrm{~mm}$ ID was used for benzene quantification. The initial column temperature was held constant for seven minutes and then programmed from $80^{\circ} \mathrm{C}$ to $140^{\circ} \mathrm{C}$ at $15^{\circ} \mathrm{C} / \mathrm{min}$. Carrier: purified helium, flow $0.8 \mathrm{ml} / \mathrm{min}$. A Hewlett Packard 5970B mass selective detector (quadrupole) was used for benzene identification.

\section{STATISTICAL ANAL YSES}

As the individual groupings were sometimes small and the distributions not always normal, the WilcoxonMann-Whitney test was used to compare the environ- 
mental, alveolar, and blood benzene concentrations among the individual groups. The Spearman test (rank correlation coefficient: rs) was used for the correlations between the environmental and biological data. The linear regression was studied when the Spearman test was statistically significant.

A test was considered statistically significant when $\mathrm{p}<0.05$.

\section{Results}

In the group of 34 workers employed in producing benzene the geometric mean of the workshift benzene exposure measured by the continuous personal sampling (Cic) was $1 \cdot 12 \mu \mathrm{g} / 1$ with a range of $0 \cdot 1-7 \cdot 4 \mu \mathrm{g} / 1$ (fig 1). In the group of 27 non-exposed workers employed in the same plant (group A) continuous personal sampling showed that the eight hour exposure was $0.1 \mu \mathrm{g} / 1$ in only four subjects. In the remaining 23 subjects of the group (group A) the eight hour exposure was indeterminable (or lower than $0 \cdot 1 \mu \mathrm{g} / \mathrm{l})$.

In accordance with the occupational exposure and the habit of smoking of the examined subjects the results of benzene determinations in the infirmary atmosphere, in the alveolar air, and blood were grouped as shown in tables 1-3. The instantaneous environmental benzene concentration measured in the plant infirmary (Ci; table 1$)$ was higher during the examination of exposed workers $(\mathrm{GM}=58 \mathrm{ng} / \mathrm{l})$ than during the examination of non-exposed workers

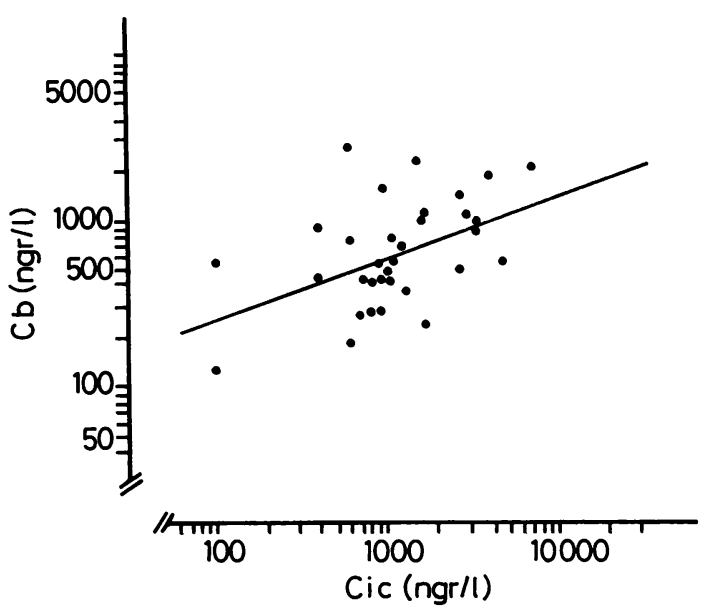

Fig 1 Correlation between workshift benzene exposure (Cic) and blood benzene concentration $(\mathrm{Cb})$ measured 16 hours after end of workshift $(\mathrm{Cb}=0.176 \mathrm{Cic}+503 ; r=$ 0.42; No = 34; $p<0.05$ ); (Cic: median $=1050 \mathrm{ng} / \mathrm{l}$; geometric mean $=1120 \mathrm{ng} / \mathrm{l} ;$ mean $=1600 \mathrm{ng} / \mathrm{l} ; S D=$ $1500 ;$ range $=100-7400)$.
Table 1 Concentrations of benzene ( $\mathrm{ng} / \mathrm{l}$ ) in environmental air (Ci) of the plant and hospital infirmaries

\begin{tabular}{|c|c|c|c|c|c|c|c|}
\hline & \multirow[b]{2}{*}{ No } & \multicolumn{6}{|c|}{$C i(n g / l)$} \\
\hline & & $M d$ & $G M$ & $M n$ & $C L$ & $S D$ & Range \\
\hline $\begin{array}{l}\text { Exposed: } \\
\text { Non-smokers } \\
\text { Smokers }\end{array}$ & $\begin{array}{l}34 \\
18 \\
16\end{array}$ & $\begin{array}{r}89 \\
112 \\
85\end{array}$ & $\begin{array}{l}58 \\
62 \\
53\end{array}$ & $\begin{array}{r}93 \\
101 \\
83\end{array}$ & $\begin{array}{l}25 \\
39 \\
33\end{array}$ & $\begin{array}{l}70 \\
78 \\
61\end{array}$ & $\begin{array}{r}2-255 \\
10-227 \\
2-255\end{array}$ \\
\hline $\begin{array}{l}\text { Non-exposed } \\
\text { (group A): } \\
\text { Non-smokers } \\
\text { Smokers }\end{array}$ & $\begin{array}{l}27 \\
11 \\
16\end{array}$ & $\begin{array}{l}38 \\
23 \\
64\end{array}$ & $\begin{array}{l}36 \\
27 \\
45\end{array}$ & $\begin{array}{l}55 \\
34 \\
70\end{array}$ & $\begin{array}{l}20 \\
15 \\
31\end{array}$ & $\begin{array}{l}50 \\
22 \\
59\end{array}$ & $\begin{array}{l}5-203 \\
5-70 \\
9-203\end{array}$ \\
\hline $\begin{array}{l}\text { Non-exposed } \\
\text { (group B): } \\
\text { Non-smokers } \\
\text { Smokers }\end{array}$ & $\begin{array}{r}19 \\
8 \\
11\end{array}$ & $\begin{array}{l}3 \\
3 \\
4\end{array}$ & $\begin{array}{l}5 \\
5 \\
5\end{array}$ & $\begin{array}{l}12 \\
13 \\
11\end{array}$ & $\begin{array}{l}10 \\
17 \\
14\end{array}$ & $\begin{array}{l}21 \\
21 \\
21\end{array}$ & $\begin{array}{l}1-75 \\
1-62 \\
2-75\end{array}$ \\
\hline $\begin{array}{l}\text { Non-exposed } \\
\quad(\text { groups A + B): } \\
\text { Non-smokers } \\
\text { Smokers }\end{array}$ & $\begin{array}{l}46 \\
19 \\
27\end{array}$ & $\begin{array}{l}19 \\
20 \\
12\end{array}$ & $\begin{array}{l}16 \\
13 \\
19\end{array}$ & $\begin{array}{l}37 \\
25 \\
45\end{array}$ & $\begin{array}{l}14 \\
12 \\
22\end{array}$ & $\begin{array}{l}46 \\
24 \\
55\end{array}$ & $\begin{array}{l}1-203 \\
1-70 \\
2-203\end{array}$ \\
\hline $\begin{array}{l}\text { All: } \\
\text { Non-smokers } \\
\text { Smokers }\end{array}$ & $\begin{array}{l}80 \\
37 \\
43\end{array}$ & $\begin{array}{l}36 \\
25 \\
54\end{array}$ & $\begin{array}{l}28 \\
27 \\
28\end{array}$ & $\begin{array}{l}60 \\
62 \\
60\end{array}$ & $\begin{array}{l}15 \\
23 \\
18\end{array}$ & $\begin{array}{l}63 \\
68 \\
60\end{array}$ & $\begin{array}{l}1-255 \\
1-227 \\
2-255\end{array}$ \\
\hline
\end{tabular}

Md, median; GM, geometric mean; Mn, mean; CL, confidence limit; $\mathrm{SD}$, standard deviation.

Table 2 Concentrations of benzene (ng/l) in the alveolar air (Ca) of the chemical workers and hospital staff

\begin{tabular}{|c|c|c|c|c|c|c|c|}
\hline & \multirow[b]{2}{*}{ No } & \multicolumn{6}{|c|}{$\mathrm{Ca}(n g / l)$} \\
\hline & & $M d$ & $G M$ & $M n$ & $C L$ & $S D$ & Range \\
\hline $\begin{array}{l}\text { Exposed: } \\
\text { Non-smokers } \\
\text { Smokers }\end{array}$ & $\begin{array}{l}34 \\
18 \\
16\end{array}$ & $\begin{array}{l}73 \\
66 \\
76\end{array}$ & $\begin{array}{l}70 \\
64 \\
76\end{array}$ & $\begin{array}{r}92 \\
83 \\
103\end{array}$ & $\begin{array}{l}27 \\
30 \\
50\end{array}$ & $\begin{array}{l}77 \\
61 \\
93\end{array}$ & $\begin{array}{l}14-377 \\
14-230 \\
16-377\end{array}$ \\
\hline $\begin{array}{l}\text { Non-exposed } \\
\text { (group A): } \\
\text { Non-smokers } \\
\text { Smokers }\end{array}$ & $\begin{array}{l}27 \\
11 \\
16\end{array}$ & $\begin{array}{l}30 \\
24 \\
42\end{array}$ & $\begin{array}{l}28 \\
17 \\
41\end{array}$ & $\begin{array}{l}41 \\
25 \\
53\end{array}$ & $\begin{array}{l}15 \\
14 \\
22\end{array}$ & $\begin{array}{l}37 \\
20 \\
41\end{array}$ & $\begin{array}{l}1-171 \\
1-77 \\
8-171\end{array}$ \\
\hline $\begin{array}{l}\text { Non-exposed } \\
\text { (group B): } \\
\text { Non-smokers } \\
\text { Smokers }\end{array}$ & $\begin{array}{r}19 \\
8 \\
11\end{array}$ & $\begin{array}{r}25 \\
2 \\
34\end{array}$ & $\begin{array}{r}14 \\
3 \\
38\end{array}$ & $\begin{array}{r}29 \\
6 \\
45\end{array}$ & $\begin{array}{r}14 \\
6 \\
18\end{array}$ & $\begin{array}{r}28 \\
8 \\
27\end{array}$ & $\begin{array}{r}1-106 \\
1-25 \\
10-106\end{array}$ \\
\hline $\begin{array}{l}\text { Non-exposed } \\
\quad(\text { groups A + B): } \\
\text { Non-smokers } \\
\text { Smokers }\end{array}$ & $\begin{array}{l}46 \\
19 \\
27\end{array}$ & $\begin{array}{l}30 \\
11 \\
41\end{array}$ & $\begin{array}{r}21 \\
9 \\
40\end{array}$ & $\begin{array}{l}36 \\
17 \\
50\end{array}$ & $\begin{array}{r}10 \\
9 \\
14\end{array}$ & $\begin{array}{l}34 \\
19 \\
36\end{array}$ & $\begin{array}{l}1-171 \\
1-77 \\
2-171\end{array}$ \\
\hline $\begin{array}{l}\text { All: } \\
\text { Non-smokers } \\
\text { Smokers }\end{array}$ & $\begin{array}{l}80 \\
37 \\
43\end{array}$ & $\begin{array}{l}43 \\
28 \\
54\end{array}$ & $\begin{array}{l}35 \\
23 \\
51\end{array}$ & $\begin{array}{l}60 \\
49 \\
69\end{array}$ & $\begin{array}{l}14 \\
19 \\
20\end{array}$ & $\begin{array}{l}62 \\
55 \\
67\end{array}$ & $\begin{array}{l}1-377 \\
1-230 \\
3-377\end{array}$ \\
\hline
\end{tabular}

Md, median; GM, geometric mean; Mn, mean; CL, confidence limit $\mathrm{SD}$, standard deviation.

(group A: GM $=36 \mathrm{ng} / \mathrm{l}$ ). In the hospital infirmaries the instantaneous benzene concentration was, as a geometric mean, $5 \mathrm{ng} / \mathrm{l}$. The differences in environmental benzene both between exposed and nonexposed individuals and between groups $A$ and $B$ were statistically significant (table 4). No statistical difference was found in the instantaneous environmental benzene concentrations between smokers and 
Table 3 Concentrations of benzene (ng/l) in blood ( $\mathrm{Cb}$ ) of the chemical workers and hospital staff

\begin{tabular}{|c|c|c|c|c|c|c|c|}
\hline & \multirow[b]{2}{*}{ No } & \multicolumn{6}{|c|}{$C b(\boldsymbol{n g} / l)$} \\
\hline & & $M d$ & $G M$ & $M n$ & $C L$ & $S D$ & Range \\
\hline $\begin{array}{l}\text { Exposed: } \\
\text { Non-smokers } \\
\text { Smokers }\end{array}$ & $\begin{array}{l}34 \\
18 \\
16\end{array}$ & $\begin{array}{l}553 \\
503 \\
556\end{array}$ & $\begin{array}{l}597 \\
607 \\
586\end{array}$ & $\begin{array}{l}789 \\
793 \\
785\end{array}$ & $\begin{array}{l}220 \\
298 \\
304\end{array}$ & $\begin{array}{l}629 \\
598 \\
683\end{array}$ & $\begin{array}{l}135-2655 \\
175-2203 \\
135-2655\end{array}$ \\
\hline $\begin{array}{c}\text { Non-exposed } \\
\text { (group A): } \\
\text { Non-smokers } \\
\text { Smokers }\end{array}$ & $\begin{array}{l}27 \\
11 \\
16\end{array}$ & $\begin{array}{l}299 \\
188 \\
367\end{array}$ & $\begin{array}{l}256 \\
196 \\
308\end{array}$ & $\begin{array}{l}307 \\
225 \\
363\end{array}$ & $\begin{array}{r}71 \\
82 \\
105\end{array}$ & $\begin{array}{l}181 \\
122 \\
197\end{array}$ & $\begin{array}{l}91-825 \\
91-438 \\
92-825\end{array}$ \\
\hline $\begin{array}{c}\text { Non-exposed } \\
\text { (group B): } \\
\text { Non-smokers } \\
\text { Smokers }\end{array}$ & $\begin{array}{r}19 \\
8 \\
11\end{array}$ & $\begin{array}{l}262 \\
127 \\
578\end{array}$ & $\begin{array}{l}269 \\
116 \\
497\end{array}$ & $\begin{array}{l}392 \\
127 \\
584\end{array}$ & $\begin{array}{r}156 \\
45 \\
201\end{array}$ & $\begin{array}{r}323 \\
54 \\
300\end{array}$ & $\begin{array}{r}49-1136 \\
49-191 \\
109-1136\end{array}$ \\
\hline $\begin{array}{c}\text { Non-exposed } \\
\text { (groups A + } \\
\text { Non-smokers } \\
\text { Smokers }\end{array}$ & $\begin{array}{r}\text { B): } 46 \\
19 \\
27\end{array}$ & $\begin{array}{l}283 \\
166 \\
458\end{array}$ & $\begin{array}{l}262 \\
157 \\
374\end{array}$ & $\begin{array}{l}342 \\
184 \\
453\end{array}$ & $\begin{array}{r}74 \\
52 \\
104\end{array}$ & $\begin{array}{l}250 \\
109 \\
263\end{array}$ & $\begin{array}{l}49-1136 \\
49-438 \\
92-1136\end{array}$ \\
\hline $\begin{array}{l}\text { All: } \\
\text { Non-smokers } \\
\text { Smokers }\end{array}$ & $\begin{array}{l}80 \\
37 \\
43\end{array}$ & $\begin{array}{l}405 \\
299 \\
465\end{array}$ & $\begin{array}{l}371 \\
303 \\
442\end{array}$ & $\begin{array}{l}532 \\
480 \\
576\end{array}$ & $\begin{array}{l}111 \\
173 \\
150\end{array}$ & $\begin{array}{l}500 \\
519 \\
485\end{array}$ & $\begin{array}{l}49-2655 \\
49-2203 \\
92-2655\end{array}$ \\
\hline
\end{tabular}

Md, median; GM, geometric mean; Mn, mean; CL, confidence limit; $\mathrm{SD}$, standard deviation. non-smokers of all the groupings considered (table 4).

The geometric mean of the alveolar benzene concentration (measured 16 hours after the end of the workshift; table $2: \mathrm{Ca}$ ) was $70 \mathrm{ng} / \mathrm{l}$ in the 34 exposed workers, $28 \mathrm{ng} / \mathrm{l}$ in group A (workers), and $14 \mathrm{ng} / \mathrm{l}$ in the hospital staff (group B). The alveolar benzene differences were statistically significant between the groups of exposed and non-exposed individuals, and between all the groups of smokers and nonsmokers, apart from the 34 exposed workers (table 4). Between groups A and B no statistically significant difference was found with regard to the alveolar benzene concentration (table 4).

The geometric mean of blood benzene concentration (table 3: $\mathrm{Cb}$ ) was $597 \mathrm{ng} / \mathrm{l}$ in the 34 exposed workers, $256 \mathrm{ng} / \mathrm{l}$ in group $\mathrm{A}$, and $269 \mathrm{ng} / \mathrm{l}$ in the hospital staff. The blood benzene differences between exposed and non-exposed individuals and between smokers and non-smokers showed a trend similar to that reported for the alveolar benzene concentrations (table 4). Generally, both the alveolar and the blood benzene concentrations were higher in the smoker than in the non-smoker groups, except for the case of the 34 exposed workers (tables 2 and 3 ).

Table 4 Results of the Wilcoxon-Mann-Whitney test on the concentrations of benzene in the infirmary air (Ci), alveolar air $(\mathrm{Ca})$, and blood $(\mathrm{Cb})$

\begin{tabular}{|c|c|c|c|c|c|c|}
\hline & \multicolumn{2}{|l|}{$C i$} & \multicolumn{2}{|l|}{$\mathrm{Ca}$} & \multicolumn{2}{|l|}{$C b$} \\
\hline & $Z$ & $p$ & $Z$ & $p$ & $Z$ & $p$ \\
\hline $\begin{array}{l}\text { Exposed: non-exposed group A } \\
\text { Exposed: non-exposed group B } \\
\text { Exposed: non-exposed group A + B } \\
\text { Group A:group B }\end{array}$ & $\begin{array}{l}2 \cdot 10 \\
4 \cdot 95 \\
4 \cdot 02 \\
4 \cdot 38\end{array}$ & $\begin{array}{l}<0.05 \\
<0.0001 \\
<0.0001 \\
<0.0001\end{array}$ & $\begin{array}{l}3 \cdot 72 \\
4 \cdot 25 \\
4 \cdot 73 \\
1 \cdot 22\end{array}$ & $\begin{array}{l}<0.0001 \\
<0.0001 \\
<0.0001 \\
\text { NS }\end{array}$ & $\begin{array}{l}3.96 \\
2 \cdot 63 \\
4 \cdot 05 \\
0 \cdot 36\end{array}$ & $\begin{array}{l}<0.0001 \\
<0.01 \\
<0.0001 \\
\text { NS }\end{array}$ \\
\hline
\end{tabular}

Table 5 Correlations between alveolar ( $\mathrm{Ca}$ ) and environmental (Cic;Ci) benzene concentrations

\begin{tabular}{|c|c|c|c|c|c|c|}
\hline & \multirow[b]{2}{*}{$Y=b x+a$} & \multirow[b]{2}{*}{$\boldsymbol{r}$} & \multirow[b]{2}{*}{ No } & \multirow[b]{2}{*}{$p$} & \multicolumn{2}{|c|}{ Spearman test } \\
\hline & & & & & $\overline{r s}$ & $p$ \\
\hline $\begin{array}{l}\text { Exposed: } \\
\text { Non-exposed groups A + B: } \\
\text { Non-smokers } \\
\text { Smokers } \\
\text { All: } \\
\text { Non-smokers } \\
\text { Smokers }\end{array}$ & $\begin{array}{l}\mathrm{Ca}=0.02 \mathrm{Cic}+66 \\
\mathrm{Ca}=0.34 \mathrm{Ci}+61 \\
\mathrm{Ca}=0.45 \mathrm{Ci}+20 \\
\mathrm{Ca}=0.45 \mathrm{Ci}+6 \\
\mathrm{Ca}=0.38 \mathrm{Ci}+33 \\
\mathrm{Ca}=0.50 \mathrm{Ci}+30 \\
\mathrm{Ca}=0.57 \mathrm{Ci}+14 \\
\mathrm{Ca}=0.42 \mathrm{Ci}+44\end{array}$ & $\begin{array}{l}0.32 \\
0.31 \\
0.61 \\
0.59 \\
0.58 \\
0.51 \\
0.71 \\
0.37\end{array}$ & $\begin{array}{l}34 \\
34 \\
46 \\
19 \\
27 \\
80 \\
37 \\
43\end{array}$ & $\begin{array}{l}\text { NS } \\
\text { NS } \\
<0.001 \\
<0.01 \\
<0.001 \\
<0.001 \\
<0.001 \\
<0.05\end{array}$ & $\begin{array}{l}0.34 \\
0.49 \\
0.52 \\
0.72 \\
0.45 \\
0.63 \\
0.74 \\
0.52\end{array}$ & $\begin{array}{l}<0.05 \\
<0.01 \\
<0.001 \\
<0.001 \\
<0.05 \\
<0.001 \\
<0.001 \\
<0.001\end{array}$ \\
\hline
\end{tabular}

Cic, environmental concentration measured continuously during workshift; $\mathrm{Ci}$, instantaneous environmental concentration measured in infirmaries. 
Table 6 Correlations between blood $(\mathrm{Cb})$ and alveolar $(\mathrm{Ca})$ benzene concentrations

\begin{tabular}{|c|c|c|c|c|c|c|c|}
\hline & \multirow[b]{2}{*}{$Y=b x+a$} & & \multirow[b]{2}{*}{$r$} & \multirow[b]{2}{*}{$\mathrm{No}$} & \multirow[b]{2}{*}{$p$} & \multicolumn{2}{|c|}{ Spearman test } \\
\hline & & & & & & $r s$ & $p$ \\
\hline $\begin{array}{l}\text { Exposed: } \\
\text { Non-exposed (groups A+B): } \\
\text { Non-smokers } \\
\text { Smokers } \\
\text { All: } \\
\text { Non-smokers } \\
\text { Smokers }\end{array}$ & $\begin{array}{l}\mathrm{Cb}=7.4 \mathrm{Ca} \\
\mathrm{Cb}=4.8 \mathrm{Ca} \\
\mathrm{Cb}=3.8 \mathrm{Ca} \\
\mathrm{Cb}=3.7 \mathrm{Ca} \\
\mathrm{Cb}=7.1 \mathrm{Ca} \\
\mathrm{Cb}=7.5 \mathrm{Ca} \\
\mathrm{Cb}=6.5 \mathrm{Ca}\end{array}$ & $\begin{array}{l}+104 \\
-141 \\
+117 \\
-120 \\
+104 \\
+108 \\
+123\end{array}$ & $\begin{array}{l}0.91 \\
0.64 \\
0.64 \\
0.50 \\
0.89 \\
0 \cdot 80 \\
0.91\end{array}$ & $\begin{array}{l}34 \\
46 \\
19 \\
27 \\
80 \\
37 \\
43\end{array}$ & $\begin{array}{l}<0.001 \\
<0.001 \\
<0.01 \\
<0.01 \\
<0.001 \\
<0.001 \\
<0.001\end{array}$ & $\begin{array}{l}0.85 \\
0.80 \\
0.66 \\
0.64 \\
0.84 \\
0 \cdot 86 \\
0 \cdot 76\end{array}$ & $\begin{array}{l}<0.001 \\
<0.001 \\
<0.01 \\
<0.001 \\
<0.001 \\
<0.001 \\
<0.001\end{array}$ \\
\hline
\end{tabular}

Table 7 Correlations between blood ( $\mathrm{Cb}$ ) and environmental ( $\mathrm{Cic}$; $\mathrm{Ci}$ ) benzene concentrations

\begin{tabular}{|c|c|c|c|c|c|c|}
\hline & \multirow[b]{2}{*}{$Y=b x+a$} & \multirow[b]{2}{*}{$r$} & \multirow[b]{2}{*}{ No } & \multirow[b]{2}{*}{$p$} & \multicolumn{2}{|c|}{ Spearman test } \\
\hline & & & & & $r s$ & $p$ \\
\hline $\begin{array}{l}\text { Exposed: } \\
\text { Non-exposed (groups A+ B): } \\
\text { Non-smokers } \\
\text { Smokers } \\
\text { All: } \\
\text { Non-smokers } \\
\text { Smokers }\end{array}$ & $\begin{array}{l}\mathrm{Cb}=0.18 \mathrm{Cic}+503 \\
\mathrm{Cb}=2.1 \mathrm{Ci}+592 \\
\mathrm{Cb}=0.7 \mathrm{Ci}-315 \\
\mathrm{Cb}=1.5 \mathrm{Ci}+147 \\
\mathrm{Cb}=-0.1 \mathrm{Ci}+458 \\
\mathrm{Cb}=2.8 \mathrm{Ci}+360 \\
\mathrm{Cb}=4.0 \mathrm{Ci}+288 \\
\mathrm{Cb}=1.5 \mathrm{Ci}+488\end{array}$ & $\begin{array}{l}0.42 \\
0.24 \\
0.13 \\
0.32 \\
0.02 \\
0.36 \\
0.53 \\
0 \cdot 18\end{array}$ & $\begin{array}{l}34 \\
34 \\
46 \\
19 \\
27 \\
80 \\
37 \\
43\end{array}$ & $\begin{array}{l}<0.05 \\
\text { NS } \\
\text { NS } \\
\text { NS } \\
\text { NS } \\
<0.01 \\
<0.001 \\
\text { NS }\end{array}$ & $\begin{array}{l}0 \cdot 48 \\
0 \cdot 34 \\
0 \cdot 17 \\
0 \cdot 36 \\
0 \cdot 16 \\
0 \cdot 39 \\
0 \cdot 59 \\
0 \cdot 12\end{array}$ & $\begin{array}{l}<0.01 \\
<0.05 \\
\text { NS } \\
\text { NS } \\
\text { NS } \\
<0.001 \\
<0.001 \\
\text { NS }\end{array}$ \\
\hline
\end{tabular}

Cic, environmental concentration measured continuously during workshift; $\mathrm{Ci}$, instantaneous environmental concentration measured in infirmaries.

Since, with reference to the biological benzene concentrations, group $\mathrm{A}$ and the non-smokers in the group of the exposed workers were not statistically different (table 4) from group B and smokers respectively, these four groups were not taken into consideration individually for the correlation analysis (tables 5-7). According to the Spearman test, all the groups considered showed a significant correlation between alveolar and environmental concentrations

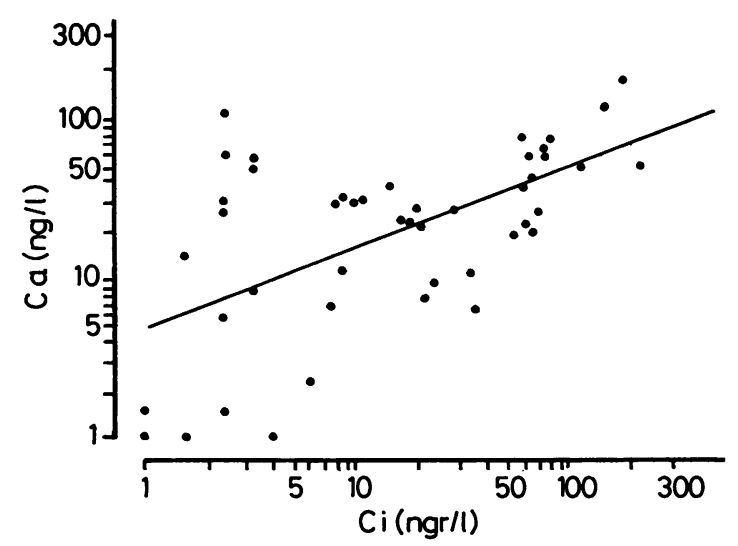

Fig 2 Correlation between alveolar ( $\mathrm{Ca}$ ) and infirmary (Ci) benzene concentrations in subjects of group $A+B$ with no occupational exposure $(\mathrm{Ca}=0.45 \mathrm{Ci}+20 ; \mathrm{r}=0.6 ; \mathrm{No}$ $=46 ; p<0.001$ ). and between blood and alveolar concentrations but not between blood and environmental benzene concentrations.

With reference to the main groups underlined in table 5 , group $A+B$ was found to show the best linear correlation between the alveolar $(\mathrm{Ca})$ and the environmental $(\mathrm{Ci})$ benzene concentrations $(r=0.61)$. The slope of the regression line between alveolar and environmental benzene concentrations in this group

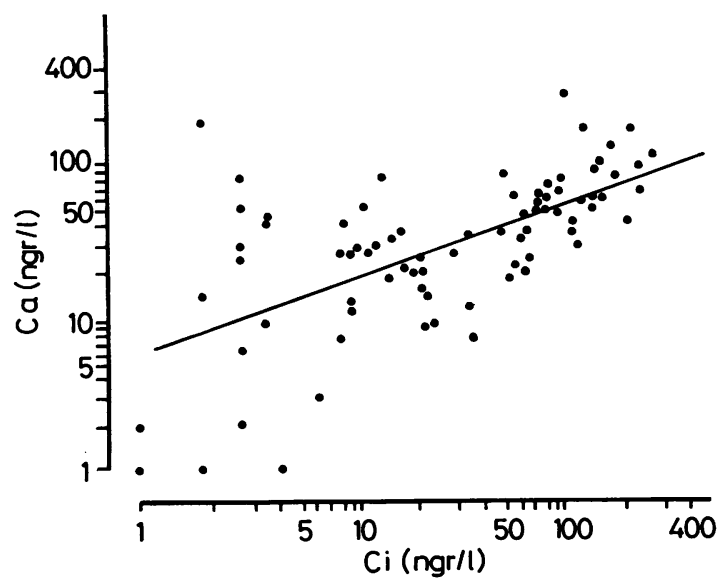

Fig 3 Correlation between alveolar ( $\mathrm{Ca}$ ) and infirmary (Ci) benzene concentrations in all the subjects studied with and without occupational exposure $(\mathrm{Ca}=0.50 \mathrm{Ci}+30 ; \mathrm{r}$ $=0.5050 ; \mathrm{No}=80 ; p<0.001)$. 


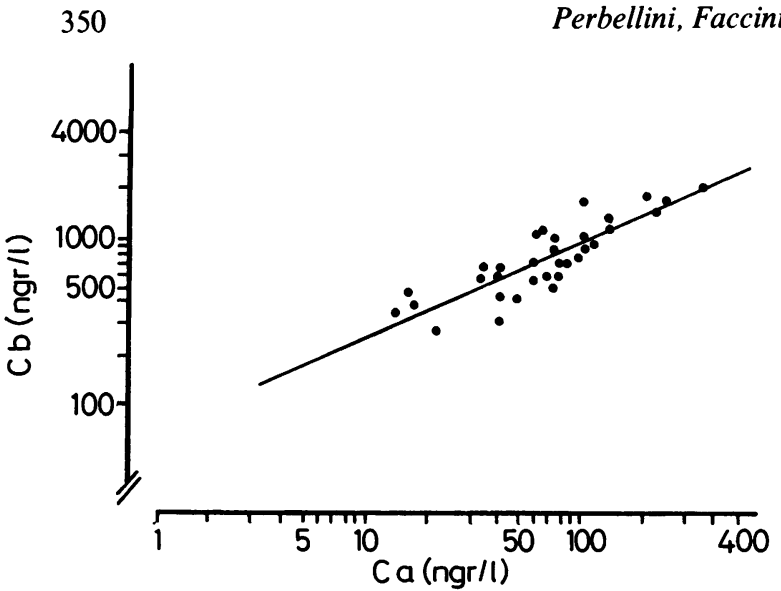

Fig 4 Correlation between blood ( $\mathrm{Cb}$ ) and alveolar ( $\mathrm{Ca}$ ) benzene concentrations in occupationally exposed workers $(C b=7.4 C a+104 ; r=0.91) ; N o=34 ; p<0.001)$.

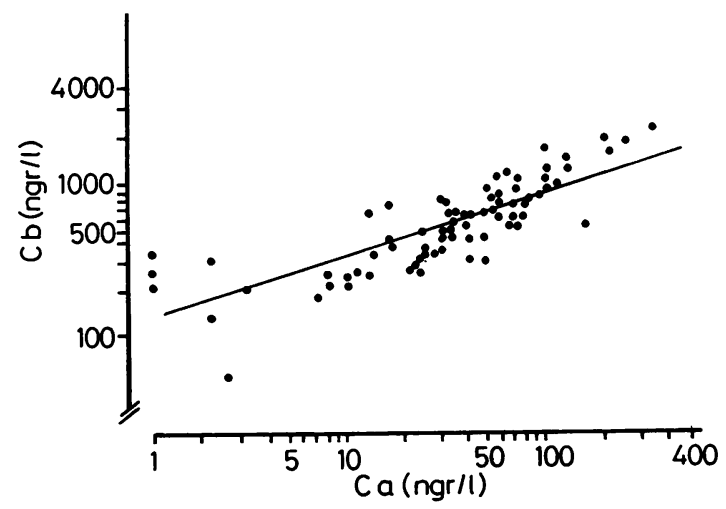

Fig 5 Correlation between blood ( $\mathrm{Cb}$ ) and alveolar ( $\mathrm{Ca}$ ) benzene concentrations in all subjects studied with and without occupational exposure $(\mathrm{Cb}=7 \cdot 1 \mathrm{Ca}+104 ; r=$ $0 \cdot 89) ;$ No $=80 ; p<0.001)$.

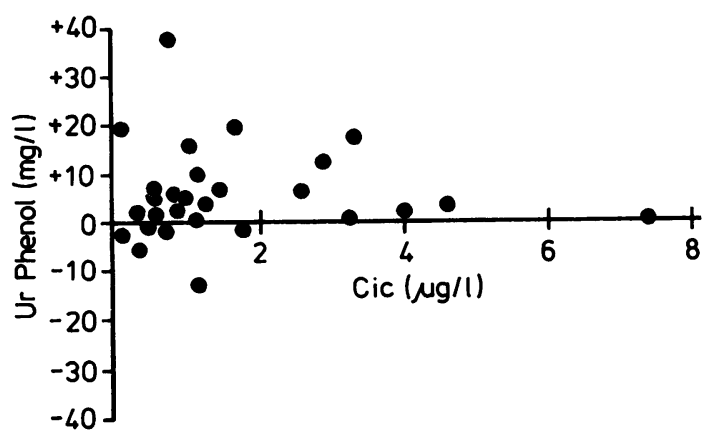

Fig 6 Relationship between workshift benzene exposure (Cic) and urinary phenol excretion (difference between excretion at end of workshift and morning after) in occupationally exposed workers.
$\mathrm{A}+\mathrm{B}$ was 0.45 , suggesting an alveolar benzene retention $(1-\mathrm{Ca} / \mathrm{Ci})$ of $55 \%$. In the group of the 34 exposed workers no linear correlation was found between alveolar benzene concentrations and the environmental benzene concentrations measured both during the workshift (Cic) and in the infirmary (Ci). Figures 2 and 3 show the correlations between alveolar and infirmary benzene concentrations in group $\mathrm{A}+\mathrm{B}$ and in all the data together.

Table 6 shows that the correlation between blood and alveolar benzene concentrations was higher in the exposed workers $(r=0.91)$ than in all the data together $(r=0.89)$ and in group $A+B(r=0.64)$. The slope of the regression line between blood and alveolar benzene concentrations, which corresponds to the $\mathrm{Cb} /$ Ca partition coefficient (solubility coefficient of benzene in blood) was $7 \cdot 4$ in the exposed workers and 4.8 in group A + B. Figures 4 and 5 show the correlations between blood and alveolar benzene concentrations in the 34 occupationally exposed workers and in all the data together.

According to the Spearman test (table 7), blood benzene concentration correlated significantly with the infirmary benzene concentrations $(\mathrm{Ci})$ in all the data together, and both with the workshift exposure (Cic) and the infirmary benzene concentration $(\mathrm{Ci})$ in the group of the 34 exposed workers. A significant linear correlation $(r=0.42)$ was found between blood and the workshift exposure (Cic) in the 34 exposed workers and between blood and infirmary benzene concentrations $(\mathrm{Ci})$ in all the data together $(r=0.36)$ and in the non-smokers $(r=0.53)$ of this grouping.

The urinary excretion of phenol in the 34 exposed workers was $12 \mathrm{mg} / \mathrm{l}(\mathrm{SD}=7)$ in the morning and 18 $\mathrm{mg} / \mathrm{l}(\mathrm{SD}=10)$ at the end of the workshift. Figure 6 shows that the urinary phenol excretion did not correlate with the mean eight hour benzene exposure. The same figure shows that the urinary excretion of phenol was, in six workers, higher in the morning than at the end of the workshift.

\section{Discussion}

Our data shows that the workshift benzene exposure (Cic), as a geometric mean value, was $1 \cdot 12 \mu \mathrm{g} / \mathrm{l}$ in the group of 34 workers who were employed in producing benzene, and practically indeterminable in the group of 27 workers (group A) not so employed. Moreover, our data show that benzene was determined in all the environmental air samples collected both in the plant infirmary and in the hospital infirmaries. Particularly, table 1 shows that the pollution of benzene was higher in the plant infirmary than in the hospital infirmaries. The presence of benzene in the plant infirmary, and especially in the hospital infirmaries, suggests that benzene must be considered a ubiquitous pollutant. 


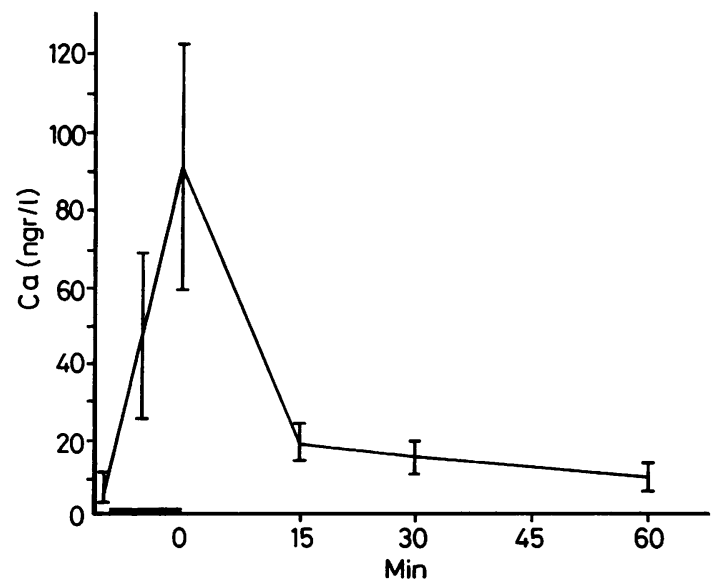

Fig 7 Variations of alveolar benzene concentration during and after smoking one cigarette in some smokers of hospital staff.

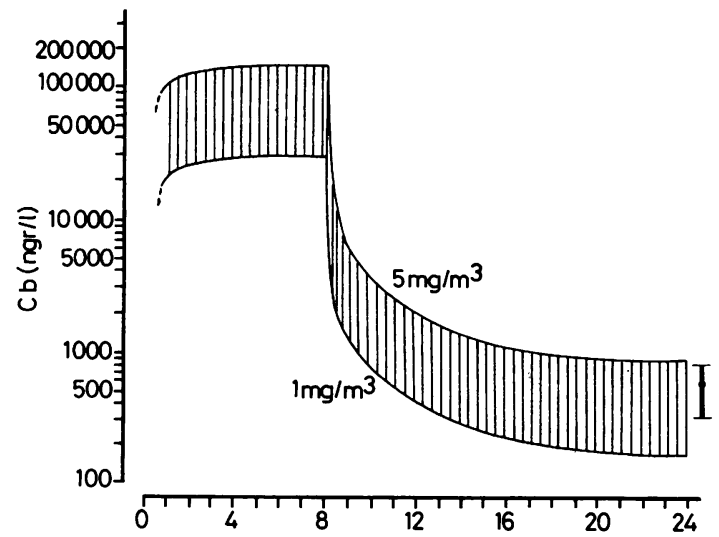

Fig 8 Simulation on mathematical model ${ }^{19}$ of variations of blood benzene concentration during and after benzene exposure of 1-5 $\mathrm{mg} / \mathrm{m}^{3}$ lasting eight hours. Thick (black) bar shows geometric mean and geometric standard deviation of blood benzene concentrations found by us in 34 chemical workers occupationally exposed to benzene.

This observation agrees with several recent studies that have reported that benzene may be detected in indoor air, outdoor air, in hospital, and in the breath of the general public. ${ }^{4-8}$ All these data suggest that the ubiquitous benzene exposure is lower than $1 \mu \mathrm{g} / 1$, more precisely in the order of $\mathrm{ng} / \mathrm{l}$. The values of 204 and $510 \mathrm{ng} / \mathrm{l}$ are the maximum benzene concentrations reported in the environmental atmosphere without any connection with occupational situations. ${ }^{48}$ Benzene concentration was found normally higher in indoor air than in outdoor air.

The alveolar and blood benzene concentrations measured in the group of the 34 exposed workers were higher (GM $=70$ and $597 \mathrm{ng} / \mathrm{l}$ respectively) than in group $A(G M=28$ and $256 \mathrm{ng} / 1)$ and group $B(G M=$ 14 and $269 \mathrm{ng} / \mathrm{l})$. Statistical analysis showed that, with reference to the alveolar and blood benzene concentrations, a significant difference existed between exposed workers and workers of group A (table 4) but not between group A and group B. The lack of a statistically significant difference between the two groups A and B suggests that these two groups have to be considered similar with reference to the benzene exposure. Since an occupational exposure was not measurable in group $\mathrm{A}$, it seems reasonable to think that the biological benzene concentrations in groups $\mathrm{A}$ and $B$ should be due to the ubiquitous benzene exposure.

It was suggested by the analysis of the data previously reported ${ }^{2}$ that an eight hour exposure to $30 \mu \mathrm{g} / 1(10 \mathrm{ppm})$ of benzene produces a benzene concentration in breath of around $360 \mathrm{ng} / 1(0 \cdot 12 \mathrm{ppm})$ 16 hours after the end of exposure. According to these data the concentration of benzene that may be found the morning after in the breath is equal to $1.2 \%$ of the exposure concentration. The geometric mean concentration of $70 \mathrm{ng} / \mathrm{l}$ of benzene found in the alveolar air of workers exposed to $1 \cdot 12 \mu \mathrm{g} / \mathrm{l}$, allow us to calculate that, 16 hours after the end of exposure, the alveolar benzene concentration was equal to $6.2 \%$ of the environmental exposure.

The alveolar and blood benzene concentrations were found to be significantly higher in the smokers than in the non-smokers of groups A and B (tables 24). It was found that the level of benzene was, generally, higher in smokers' homes than in nonsmokers. ${ }^{8}$ These findings agree with the high content of benzene in cigarette smoke. ${ }^{9-11}$ For demonstrative purposes we show in fig 7 the variations of the alveolar benzene concentrations during and after the smoking of one cigarette in some smokers of the hospital staff.

In the smokers of the group of the 34 exposed workers the alveolar and blood benzene concentrations did not statistically differ from those found in the non-smokers (table 4). These findings suggest that in the group of exposed workers the smoking effect on the biological benzene concentrations disappears because it is overwhelmed by the level of the occupational benzene exposure. Since the smoking effect did not disappear in groups A and B, it may be assumed that the effect of smoking on the alveolar and blood benzene concentrations is visible and recognisable when the environmental benzene exposure is low, but it is unrecognisable when the benzene pollution increases. An examination of the data in table 5 shows that in the group of 34 exposed workers the alveolar benzene concentration did not correlate with the infirmary benzene concentration, whereas in the nonexposed subjects (group A + B) it correlated with the infirmary benzene concentration $(r=0.61)$. Moreover, the data in table 6 show that the alveolar 
benzene concentration correlated better with the blood benzene concentration in the 34 exposed workers $(r=0.91)$ than in the non-exposed group $A+B(r=0.64)$. These results seem to suggest that the alveolar benzene concentration reflects the infirmary benzene pollution in group $\mathrm{A}+\mathrm{B}$. On the other hand, the fact that in the 34 exposed workers the alveolar benzene concentration did not correlate with the infirmary benzene concentration but, on the contrary, correlated highly with the blood benzene concentration suggests that the alveolar benzene concentration in the 34 workers reflects mainly the previous afternoon exposure and the body burden of benzene in the tissues and not the infirmary benzene pollution.

In the non-exposed group A + B (table 5 and fig 2) the slope of the regression line between alveolar and infirmary benzene concentrations was 0.45 , suggesting an alveolar benzene retention of $55 \%$. Previous data suggest a pulmonary benzene retention of $47-50 \% .{ }^{12-15}$

The correlation between blood and alveolar benzene concentrations in the 34 exposed workers (table 6 and fig 4) showed that the slope of the regression line, which corresponds to the ratio between the blood and the alveolar concentrations $(\mathrm{Cb} / \mathrm{Ca})$, was equal to $7 \cdot 4$. It is interesting to note that the blood/alveolar partition coefficient of the benzene, experimentally determined "in vitro," was found to be equal to 6.4 7.84 that is, similar to that found by us (7.4).

Finally, our results show that occupational exposure to benzene (Cic) correlated with blood benzene concentration (fig 1 and table 7) but not with alveolar benzene concentration (table 5). According to the slope of the regression line between blood concentration and workplace exposure (fig 1) the benzene concentration found in the blood the morning after turns out to be $17.6 \%$ of the previous workshift exposure. Figure 8 shows the blood benzene concentrations determined according to a mathematical model ${ }^{19}$ during and after an eight hour environmental exposure of $1-5 \mu \mathrm{g} / \mathrm{l}$. As may be seen in this figure, 16 hours after the end of the exposure the blood benzene concentration is $10-20 \%$ of the benzene exposure considered. This result is similar to that $(17 \cdot 6 \%)$ obtained by us in the occupationally exposed workers. No correlation was found between workshift benzene exposure and urinary phenol excretion measured both in the morning and at the end of the workshift (fig 6). As regards the urinary phenol excretion it is already well known that benzene exposure lower than $5 \mathrm{ppm}$ $(15 \mu \mathrm{g} / \mathrm{l})$ produces an amount of phenol that may be overwhelmed inside the upper limit of the urinary excretion $(20 \mathrm{mg} / \mathrm{l})$ due to the physiological metabolism. ${ }^{20}$

We thank the workers at the chemical plant and the hospital staff who generously cooperated and gave alveolar and blood samples in order that this investigation could be accomplished. We are also grateful to the technical staff of the Environment Protection
Service, Gaschromatography Laboratory, Clinical Chemistry Laboratory, and plant infirmary of Montedipe in Mantova for their cooperation.

This work was in part supported by Italian National Research Council (CNR), finalised project on preventive and rehabilitative medicine, subproject SP5: toxicological risk (grant No 85.00470.56).

\section{References}

1 International Agency for Research on Cancer. Monographs on the evaluation of the carcinogenic risk of chemicals to humans. Vol 29. Lyon: IARC, 1982:99-148.

2 Lauwerys R. Industrial health and safety: human biological monitoring of industrial chemicals series. Benzene. Luxembourg: Commission of the European Communities, 1983:18-25.

3 National Institution for Occupational Safety and Health. Manual of analytical methods. 2nd ed. Vol III. Standards completion program validated methods. Cincinnati: DHEW (NIOSH), 1977.

4 De Bortoli M, Knoppel H, Pecchio E, et al. Measurements of indoor air quality and comparison with ambient air: a study on 15 homes in northern Italy. Luxembourg: Commission of the European Communities, 1985.

5 Gage JC, Lagesson V, Tunek A. A method for the determination of low concentrations of organic vapours in air and exhaled breath. Ann Occup Hyg 1977;20:127-34.

6 Holmberg B, Lundberg P. Benzene: standards, occurrence, and exposure. Am J Ind Med 1985;7:375-83.

7 Krotoszynski BK, Bruneau GM, O'Neill HJ. Measurement of chemical inhalation exposure in urban population in the presence of endogenous effluents. J Anal Toxicol 1979;3:225-34.

8 Wallace LA, Pellizzari ED, Hartwell TD, Sparacino CM, Sheldon LS, Zelon H. Personal exposures, indoor-outdoor relationships, and breath levels of toxic air pollutants measured for 355 persons in New Jersey. Atmospheric Environment 1985;19: 1651-61.

9 Berlin M, Gage JC, Gulberg B, et al. Breath concentration as an index of the health risk from benzene. Studies on the accumulation and clearance of inhaled benzene. Scand J Work Environ Health 1980;6:104-11.

10 Berlin M. Low level benzene exposure in Sweden: effect on blood elements and body burden of benzene. Am J Ind Med 1985;7:365-73.

11 Egle J Jr, Gochberg BJ. Respiratory retention of inhaled toluene and benzene in the dog. J Toxicol Environ Health 1976;1:531-5.

12 Badodej Z. A study on absorption, metabolism and excretion of toxic vapours. Acta Universitatis Carolinae Medica supl 1964;19:47-54.

13 Fiserova-Bergerova V, Vlach J, Singhal K. Simulation and prediction of uptake, distribution, and exhalation of organic solvents. Br J Ind Med 1974;31:45-52.

14 Hunter CG, Blair D. Benzene pharmacokinetic studies in man. Ann Occup Hyg 1972;15:193-9.

15 Srbova J, Teisinger J, Skramovsky S. Absorption and elimination of inhaled benzene in man. Arch Ind Hyg Occup Med 1950;2: 1-8.

16 Fiserova-Bergerova V, Diaz LM. Determination and prediction of tissue-gas partition coefficients. Int Arch Occup Environ Health 1986;58:75-87.

17 Sato A, Nakajima T. Partition coefficients of some aromatic hydrocarbons and ketones in water, blood, and oil. Br J Ind Med 1979;36:231-4.

18 Sherwood RJ. Ostwald solubility coefficients of some industrially important substances. Br J Ind Med 1976;33:106-7.

19 Perbellini L, Mozzo P, Brugnone F, Zedde A. Physiologicomathematical model for studying human exposure to organic solvents: kinetics of blood/tissue $n$-hexane concentrations and of 2,5-hexanedione in urine. Br J Ind Med 1986;43:760-8.

20 Roush GJ, Ott MG. A study of benzene exposure versus urinary phenol levels. Am Ind Hyg Ass J 1977;38:67-75. 